\title{
Effects of Two-step Annealing on Texture and Magnetic Properties by Addition of Co, Mo, or Ni in Silicon Steel
}

\author{
Yan Bo CAI and Sam Kyu CHANG \\ Graduate Institute of Ferrous Technology, POSTECH, Hyoja-Dong, Pohang, 790-784, Korea.
}

(Received on July 13, 2007; accepted on August 22, 2007)

\begin{abstract}
In 3Si-1.4Al electrical steel with addition of $\mathrm{Co}$, Mo or $\mathrm{Ni}$, the texture of hot bands is almost random except $\gamma$ fiber which is dominant. After cold reduction, the texture shows the typical (111)//ND and (110)//RD fibers. When cold rolled sheets are annealed, it is clear that $\gamma$ fiber of $\{111\}\langle 110\rangle$ and $\{111\}\langle 112\rangle$ decreases significantly, while, on the contrary, Goss texture increases but cube texture is not changed. Two-step annealing process was observed to be effective to increase grain size and decrease $\gamma$ fiber texture resulting in lowering iron loss and its anisotropy. The combination of increasing grain size and decreasing $\gamma$ texture by two-step annealing was able to improve magnetic properties.
\end{abstract}

KEY WORDS: non-oriented electrical steel; iron loss; magnetic flux density; texture factor.

\section{Introduction}

Non-oriented electrical steel sheets have been widely used as core materials in rotating machines in which rotational magnetic fields exist. ${ }^{1)}$ The magnetic behavior of non-oriented electrical steels is controlled primarily by several microstructural features of texture, grain size, and impurity. The important structure-dependent magnetic properties are permeability and watt loss which are expected to depend on grain size and texture of the fully processed nonoriented electrical steels. ${ }^{2,3)}$ Texture evolution has attracted more academic interests. Silicon steel sheets with (100)[001] cubic texture have high permeability both in the rolling direction and the transverse direction, so that they are expected to improve the efficiency of rotating motors. Nevertheless, there is no commercial method able to attain that texture. So, the usual method for texture optimization in non-oriented electrical steels is to control grains to have orientations such as (100)[uvw] or Goss orientation with easy magnetization but to suppress orientations like (111) $[u v w]$ and (211) $[u v w]$ with hard magnetization direction.

It has been reported ${ }^{4)}$ that, for a given silicon content, core loss value in the steel initially decreases with the increase in the ferrite grain size. In industrial practice, ferrite grain size of non-oriented electrical steel is increased by adopting suitable hot strip rolling parameters which include low finishing and high coiling temperatures. Further to this, both hot rolled and cold rolled coils are required to be annealed to promote coarsening of ferrite grain size. Therefore, the present work tried to enlarge grain size and thus to gain useful textures by two-step annealing. Annealing was raised to the commercial temperature at the first step and subsequently heated to higher temperature at the second step, in which process grain will grow well by increase of thermal energy and thus growth of Goss texture is expected. The effect of two-step annealing on texture evolution and magnetic properties of high grade non-oriented electrical steels was examined and the correlation of texture factor and grain size with magnetic properties was verified.

\section{Experimental Procedure}

Test steels were melted in a vacuum furnace and their chemical compositions were shown in Table 1, in which Sn and $\mathrm{Sb}$ were basically added to improve magnetic properties. Furthermore, in order to maximize the magnetic properties, more special elements of $\mathrm{Co}$, Mo, and Ni were added to this basic alloy and their effects on the magnetic properties were investigated according to annealing conditions. The ingot were hot rolled to a thickness of $2.6 \mathrm{~mm}$. Hot rolled bands were normalized at $1000^{\circ} \mathrm{C}$ for $3 \mathrm{~min}$, and

Table 1. Chemical composition in wt \%.

\begin{tabular}{|c|c|c|c|c|c|c|c|c|c|c|c|}
\hline Samples & $\mathrm{C}$ & $\mathrm{Si}$ & $\mathrm{Mn}$ & $\mathrm{Al}$ & $\mathrm{Sn}$ & $\mathrm{Sb}$ & $\mathrm{P}$ & $\mathrm{S}$ & $\mathrm{Co}$ & $\mathrm{Mo}$ & $\mathrm{Ni}$ \\
\hline Steel A & 0.005 & 2.96 & 0.31 & 1.52 & 0.10 & 0.08 & 0.004 & 0.003 & & & \\
\hline Steel B & 0.004 & 2.91 & 0.31 & 1.52 & 0.10 & 0.08 & 0.004 & 0.003 & 0.05 & & \\
\hline Steel C & 0.004 & 2.92 & 0.31 & 1.52 & 0.10 & 0.08 & 0.004 & 0.003 & & 0.05 & \\
\hline Steel D & 0.004 & 2.92 & 0.31 & 1.51 & 0.11 & 0.08 & 0.004 & 0.003 & & & 0.05 \\
\hline
\end{tabular}


then cooled to $250^{\circ} \mathrm{C}$ at a cooling rate of $200^{\circ} \mathrm{C} / \mathrm{min}$. The sheets were finally air-cooled to room temperature. The normalized hot bands were cold rolled from $2.6 \mathrm{~mm}$ down to $0.5 \mathrm{~mm}$ in thickness. The cold rolled samples were annealed in two ways, as illustrated in Fig. 1. Single-step annealing (AFA1) was heated to $900^{\circ} \mathrm{C}$ at a reheating rate of $20^{\circ} \mathrm{C} / \mathrm{s}$, soaked for $3 \mathrm{~min}$, cooled to $300^{\circ} \mathrm{C}$ in $3 \mathrm{~min}$, and then slowly cooled to room temperature. The other way of two-step annealing (AFA2) was heated to $900^{\circ} \mathrm{C}$ at a reheating rate of $20^{\circ} \mathrm{C} / \mathrm{s}$, soaked for $1 \mathrm{~min}$, then reheated to $1050^{\circ} \mathrm{C}$ at the same reheating rate, soaked for $2 \mathrm{~min}$, and finally cooled to room temperature. The annealing atmosphere consists of $30 \% \mathrm{H}_{2}$ and $70 \% \mathrm{~N}_{2}$ to obtain a smooth surface without oxidization. This step annealing was based on the presumption to improve magnetic properties by acceleration of grain growth.

Grain size measurement was obtained by conventional metallography and image analysis. At least 150 grains were measured at random by using standard linear intercept method. The average grain size after hot rolling and final annealing was estimated. Texture analysis was carried out by X-ray diffractometry and (110), (200) and (211) pole figures were determined in the one fourth thickness of the sample to ensure uniformity in the texture distribution. Furthermore, orientation distribution function (ODF) was calculated from pole figure data. Specific textures and fibers were analyzed from the ODF data. The volume ratio of the sum of cube and Goss textures to $\gamma$ fiber was expressed as a texture factor ${ }^{5)}$ which could be well related to magnetic properties. Magnetic properties of magnetic flux density $\left(B_{50}\right)$ and iron loss $\left(W_{15 / 50}\right)$ were measured by a single sheet tester.

\section{Results}

Average grain sizes of hot bands and annealed sheets are shown in Table 2. In hot bands, grain size reveals a little

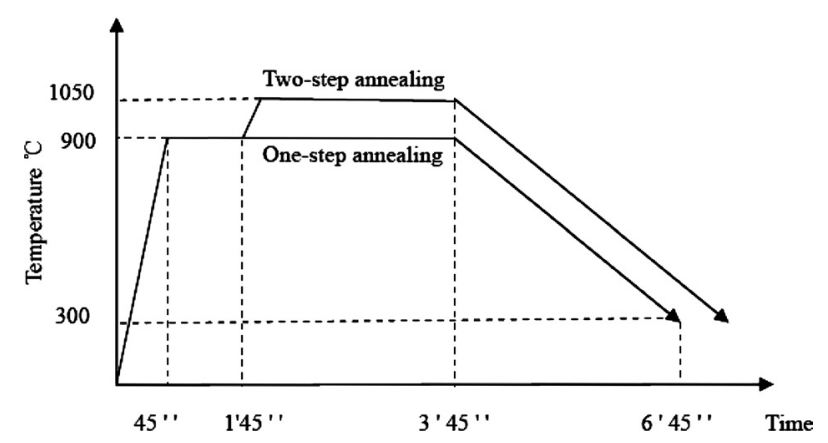

Fig. 1. Recrystallization annealing cycles. difference among all the test steels but steel $\mathrm{D}$ bearing $\mathrm{Ni}$ shows largest grain. After annealing process, average grain size increased remarkably and two-step annealing shows larger grain size than one-step annealed samples. Also Ni steel grain is largest in annealed specimens. An apparent fact indicates that addition of $\mathrm{Co}, \mathrm{Mo}$, and $\mathrm{Ni}$ results in less increment of grain size than steel A with no special elements comparing between one-step and two-step annealing. Steel B with Co is most effective to restrict the grain growth during two-step annealing.

The textures of hot bands and cold rolled specimens were compared. Figure 2 shows ODFs of hot bands and cold rolled specimens. In hot bands (Fig. 2(a)), steel A shows strong (111)[011] orientation but the other steels show very weak (111)[011] orientation and instead, very strong

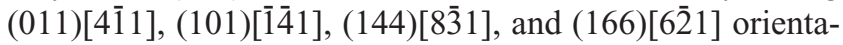
tions. Figure 2(b) shows typical cold rolling textures. The textures of the cold rolled sheets contain generally main fiber components of (110)//RD and (111)//ND. $\{111\}\langle 112\rangle$ orientation appears more sharply as well as $\{111\}\langle 110\rangle$ orientation also turns up distinctly. However, in the present specimens, steels A and B show strong (111)[0111] and (211)[011] orientations but steels C and D show another

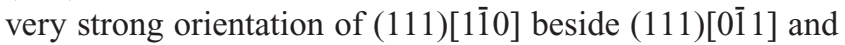
(211)[01ㅣ orientations. Therefore, Co containing steel has not a strong (111)[110] orientation differently from Mo and Ni steels. Table 3 shows quantitative volume fractions of textures. Cold rolled sheets show that Goss texture decreased but cube and $\gamma$ fiber remarkably increased comparing with hot rolled bands. Rotated cube texture of (001)[110] increased three times hot bands in volume fraction, which is a noticeable result. Figure 3 clearly shows the intensity of orientations along $\alpha$ fiber and $\gamma$ fiber and noticeable texture changes. In cold rolled sheets, $\alpha$ fiber is relatively higher intensity through $\Phi=0-70^{\circ}$ than that of hot band showing quite low intensity and also shows a peak at $\Phi=45^{\circ}$ in between $\{112\}\langle 110\rangle$ and $\{111\}\langle 110\rangle$. Also $\gamma$ fiber shows strong intensity after cold rolling and $\{111\}\langle 110\rangle$ orientation at $\varphi_{1}=60^{\circ}$ is dominant comparing with hot band.

Cold rolled specimens received different annealing processes, single-step annealing and two-step annealing. After different step annealing, the textures of these sheets were measured on one fourth thickness of specimens. The orientation distribution functions were calculated from the (200), (110) and (211) pole figures. The volume fractions of important texture components calculated from the ODFs are shown in Table 4. From hot rolling to cold rolling, as mentioned above, texture measurements showed an increase of cube texture and $\gamma$ fiber but large decrease of Goss tex-

Table 2. Grain size of hot band and annealed steels.

\begin{tabular}{|c|c|c|c|}
\hline \multirow{2}{*}{ Steel } & \multicolumn{3}{|c|}{ Mean grain size $(\mu \mathrm{m})$} \\
\cline { 2 - 4 } & Hot band & & $\begin{array}{c}\text { Two-step annealing } \\
\text { One-step annealing }\end{array}$ \\
\hline Steel A & 77.4 & 102.3 & $118.7(16 \%)$ \\
\hline Steel B & 76.4 & 112.0 & $121.3(8.3 \%)$ \\
\hline Steel C & 78.5 & 103.5 & $117.9(13.9 \%)$ \\
\hline Steel D & 81.8 & 116.5 & $128.7(10.5 \%)$ \\
\hline
\end{tabular}



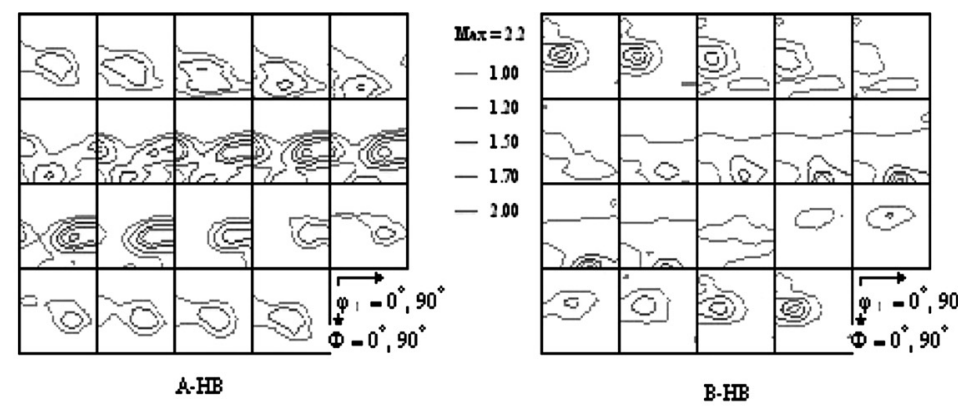

Whx $=42$

$-1.00$

$-1.60$

$-230$

$-290$

$-3.60$

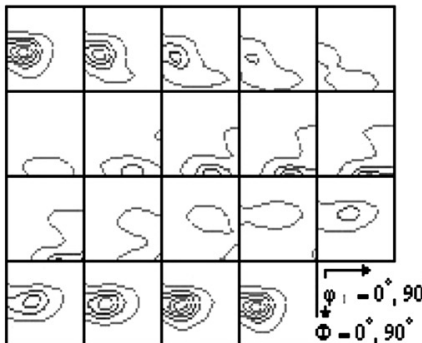

C-HB

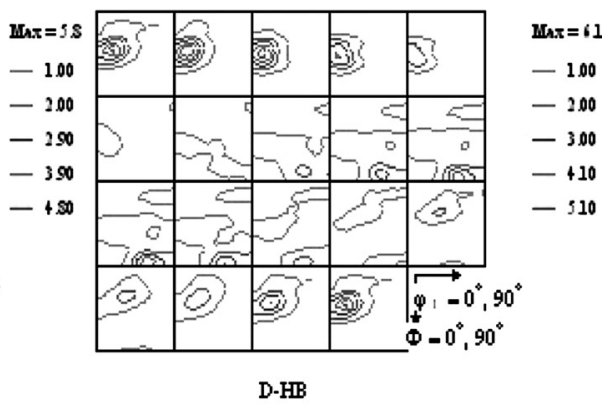

(a)
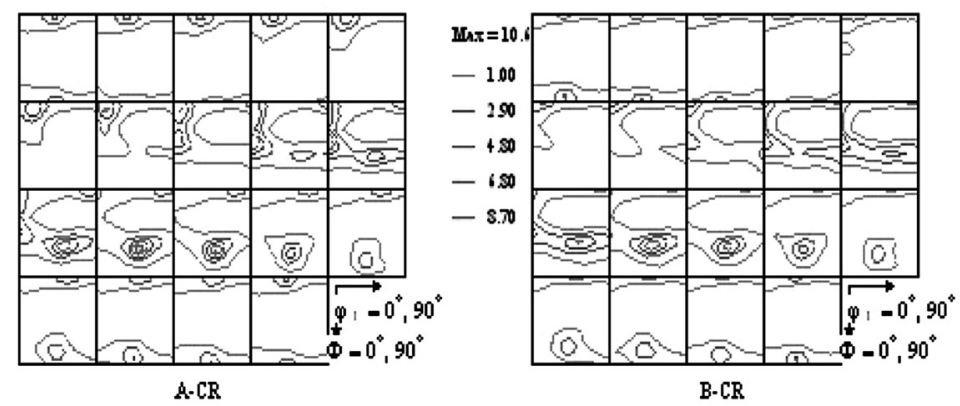

$\mathrm{max}=90$

$-100$

$-2.60$

$-420$

$-930$

$-7.40$
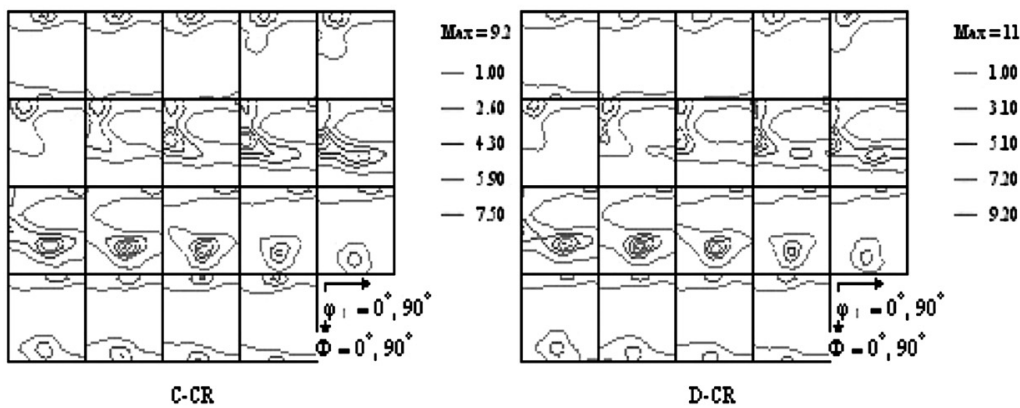

(b)

Fig. 2. ODFs of (a) hot band and (b) cold rolled specimens.

Table 3. Texture volume fractions of hot rolled bands and cold rolled sheets.

\begin{tabular}{|c|c|c|c|c|c|c|c|c|c|}
\hline \multirow{3}{*}{ Texture } & \multirow{3}{*}{ 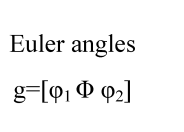 } & \multicolumn{8}{|c|}{ Texture volume (\%) } \\
\hline & & \multicolumn{2}{|c|}{ Steel A } & \multicolumn{2}{|c|}{ Steel B } & \multicolumn{2}{|c|}{ Steel C } & \multicolumn{2}{|c|}{ Steel D } \\
\hline & & $\mathrm{HB}$ & $\mathrm{CR}$ & $\mathrm{HB}$ & $\mathrm{CR}$ & $\mathrm{HB}$ & $\mathrm{CR}$ & $\mathrm{HB}$ & $\mathrm{CR}$ \\
\hline Cube & {$\left[\begin{array}{lll}0 & 0 & 0\end{array}\right]$} & 1.7 & 2.8 & 1.6 & 2.9 & 1.4 & 2.9 & 1.7 & 2.6 \\
\hline Goss & {$\left[\begin{array}{llll}90 & 90 & 45\end{array}\right]$} & 2.4 & 1.2 & 3.6 & 1.2 & 5.5 & 1.3 & 5.3 & 1.2 \\
\hline$\gamma$ fiber & {$\left[\begin{array}{llll}0-90 & 55 & 45\end{array}\right]$} & 27.3 & 50.1 & 24.2 & 49.5 & 17.9 & 48.9 & 21.1 & 54.6 \\
\hline$(001)[110]$ & {$\left[\begin{array}{lll}0 & 0 & 45\end{array}\right]$} & 1.7 & 5.8 & 1.4 & 5.3 & 1.4 & 5.3 & 1.7 & 5.2 \\
\hline \multicolumn{2}{|c|}{ (100)[uvw] } & 8.9 & 16.9 & 8.1 & 16.8 & 7.7 & 16.5 & 8.8 & 15.5 \\
\hline \multicolumn{2}{|c|}{ Texture factor $(\%),($ cube + Goss $) / \gamma$} & 15.0 & 8.0 & 21.5 & 8.3 & 38.5 & 8.6 & 33.2 & 7.0 \\
\hline
\end{tabular}



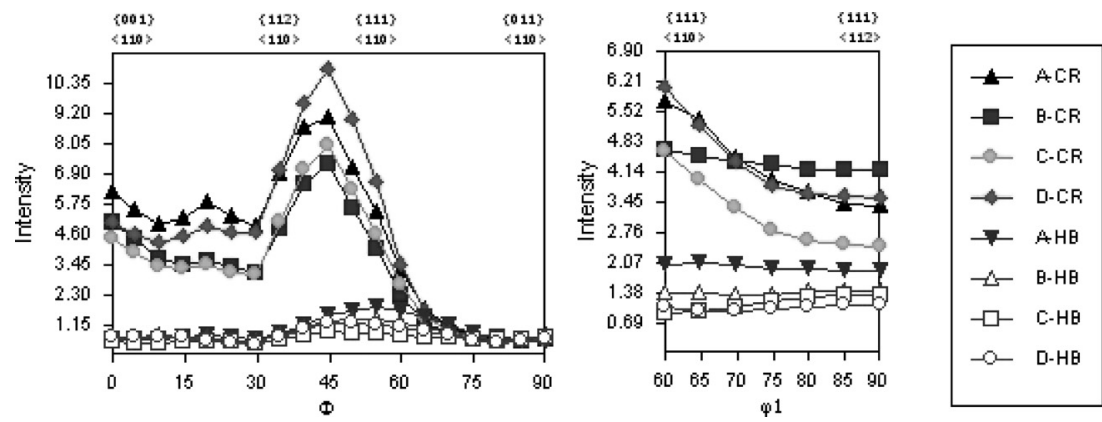

Fig. 3. Orientation densities of cold sheets as a function of $\Phi$ along $\alpha$ fiber and $\Phi 1$ along $\gamma$ fiber and noticeable texture changes from hot bands to cold sheets.

Table 4. Texture volume fractions of two annealing processes.

\begin{tabular}{|c|c|c|c|c|c|c|c|c|c|}
\hline \multirow{3}{*}{ Texture } & \multirow{3}{*}{$\begin{array}{c}\text { Euler angles } \\
\mathrm{g}=\left[\begin{array}{ll}\varphi_{1} \varphi & \varphi_{2}\end{array}\right]\end{array}$} & \multicolumn{8}{|c|}{ Texture volume (\%) } \\
\hline & & \multicolumn{2}{|c|}{ Steel A } & \multicolumn{2}{|c|}{ Steel B } & \multicolumn{2}{|c|}{ Steel C } & \multicolumn{2}{|c|}{ Steel D } \\
\hline & & FA1 & FA2 & FA1 & FA2 & FA1 & FA2 & FA1 & FA2 \\
\hline Cube & {$\left[\begin{array}{lll}0 & 0 & 0\end{array}\right]$} & 2.7 & 2.9 & 2.9 & 3.0 & 2.9 & 3.1 & 3.1 & 2.9 \\
\hline Goss & [90 9045 4 & 2.6 & 2.5 & 1.8 & 2.0 & 1.9 & 1.9 & 2.3 & 2.5 \\
\hline$\gamma$ fiber & {$\left[\begin{array}{lll}0-90 & 55 & 45\end{array}\right]$} & 27.4 & 26.8 & 31.3 & 31.3 & 31.8 & 34.4 & 25.4 & 25.0 \\
\hline$(001)[110]$ & {$\left[\begin{array}{lll}0 & 0 & 45\end{array}\right]$} & 3.4 & 3.2 & 3.3 & 3.3 & 3.2 & 3.3 & 3.5 & 3.3 \\
\hline \multicolumn{2}{|c|}{$(100)[\mathrm{uvw}]$} & 16.5 & 16.2 & 17.0 & 17.1 & 16.7 & 17.4 & 17.3 & 16.9 \\
\hline \multicolumn{2}{|c|}{ Texture factor(\%), (cube+Goss) $/ \gamma$} & 19.0 & 20.1 & 15.0 & 16.0 & 15.1 & 14.5 & 21.3 & 21.6 \\
\hline
\end{tabular}

Note: FA1 is single-step annealing and FA2 two-step annealing.

ture after cold rolling of hot bands. However, difference of each texture volume fraction between test steels is not noticeable. On the other hand, in regard of the texture development of test steels by annealing method, cube, Goss and $\gamma$ fiber texture volumes show little difference between single-step and two-step annealing. However, Goss texture apparently increased and $\gamma$ fiber decreased remarkably from cold rolling to annealing. For core materials using motors, the ideal texture is a uniform (100)//ND texture with no anisotropy. Goss texture is the ideal texture for grain-oriented electrical steels and it is very attractive component in magnetic materials since it has the highest permeability in $\langle 100\rangle$ direction parallel to the rolling direction and an intermediate permeability in $\langle 110\rangle$ direction. It is obvious that approaching randomly textured state is realized by decreasing (111) texture. Thus in order to formulate the effectiveness of textures quantitatively on magnetic properties, a texture factor was introduced and expressed as the ratio of (cube texture + Goss texture) $/ \gamma$ fiber. In the present study, $\gamma$ fiber was represented as the sum of four major orientations

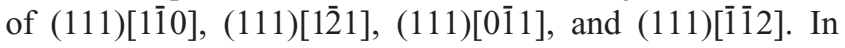
annealed sheets, the texture factor of steel $\mathrm{D}$ is highest among the test steels but there is no apparent difference in the texture factor between one-step and two-step annealing. Also strong (211) [uvw] orientation is seen and rotated cube of (001)[110] is noticeable.

Figure 4 shows orientation intensities along $\alpha, \gamma$ and $\eta$ fiber in two types of annealing processes. Typical rolling textures of $\alpha$ fiber, (110)//RD component and $\gamma$ fiber, (111)//ND component are very important factors for controlling magnetic properties. $\eta$ fiber, (100)//RD component, is a key factor for getting random texture, so called isotropic texture. Viewing the effect of two-step annealing on these fiber texture components, steel D with Ni shows lower $\gamma$ fiber for two-step annealing than for single-step annealing but shows similar intensities for $\alpha$ and $\eta$ fibers between both processes. However, Mo containing steel $\mathrm{C}$ is contrary to the Ni steel, that is, $\gamma$ fiber is higher for twostep annealing. Steel B with Co shows little difference in fiber intensities between two annealing processes. Thus the fact that two-step annealing is beneficial to decrease $\gamma$ fiber and become a practicable method for decreasing (111) component at least for Ni bearing steel was verified.

Table 5 shows the magnetic properties of final annealed sheets. Two-step annealing process shows a high magnetic flux density only in steel D but other steels show a low magnetic flux density, comparing with the one-step annealing. Differently from the magnetic flux density, iron loses are much lowered by double step annealing comparing to the single step annealing. Among the test steels, nickel added steel reveals remarkably lower iron loss than cobalt or molybdenum bearing steel. In regard of magnetic anisotropy, it is different depending upon elements added and annealing method. For steels A and D, anisotropies of magnetic induction and iron loss appear higher after twostep annealing than one-step annealing, but steels B and C shows less anisotropies after two-step annealing. The $\mathrm{Ni}$ steel showing the lowest iron loss reveals largest anisotropies in both magnetic flux density and iron loss in two-step annealing is of interest.

\section{Discussion}

Comparing annealed sheets between single-step and twostep annealing, it was evidenced that the two-step annealing process was effective to get desirable cube and Goss tex- 

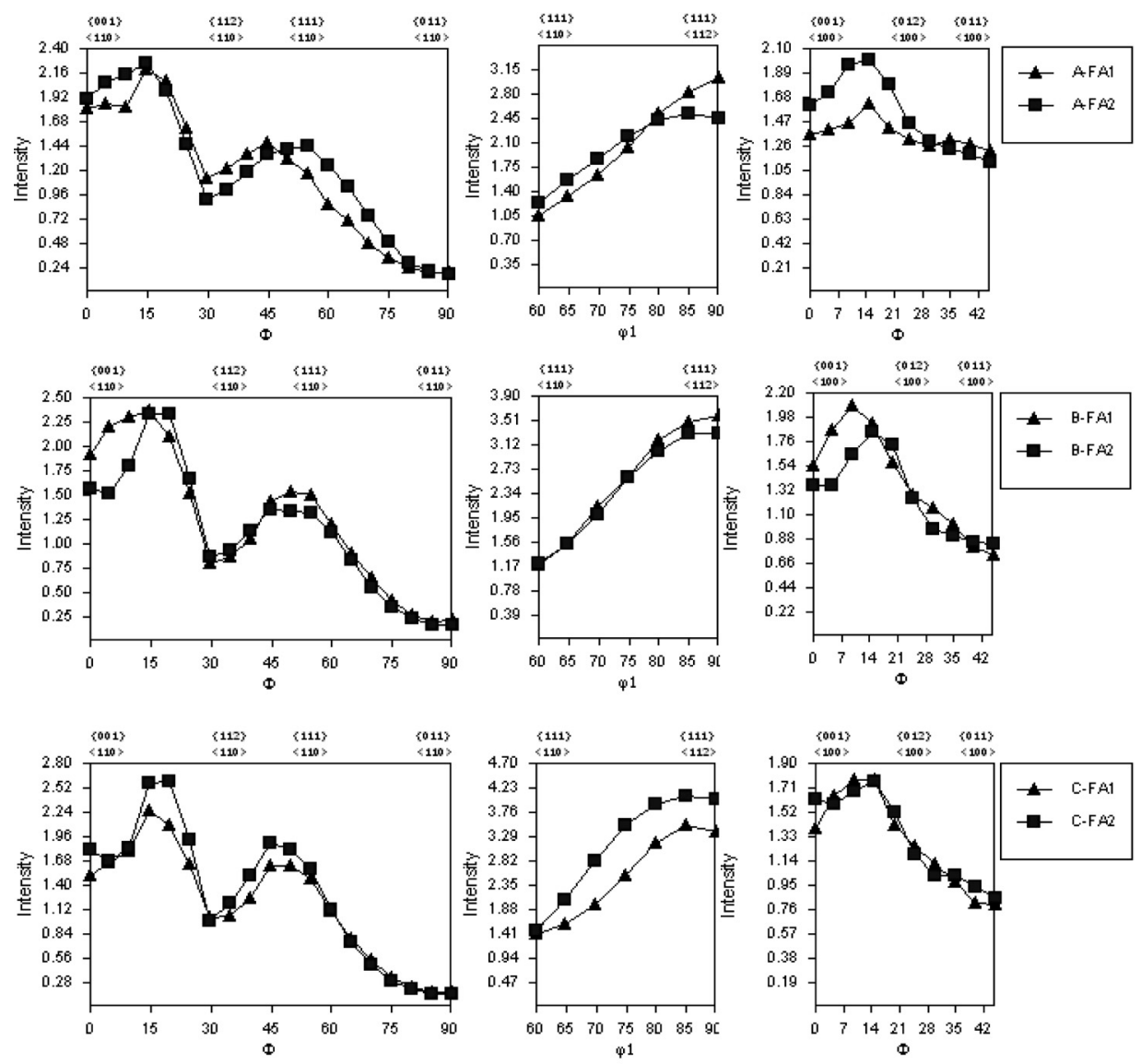

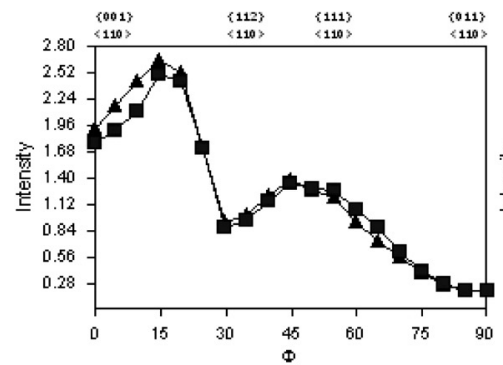

(a)

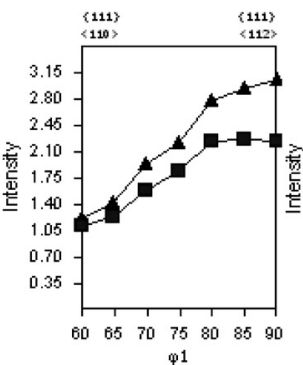

(b)

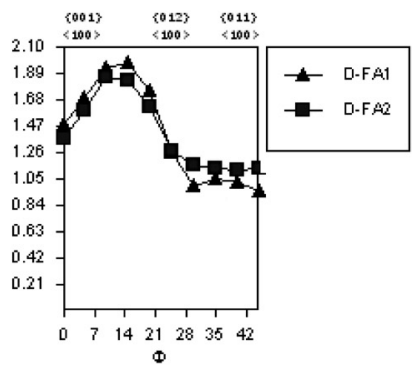

(c)

Fig. 4. Orientation intensities of two types of annealing as a function of (a) $\Phi$ along $\alpha$ fiber, (b) $\phi_{1}$ along $\gamma$ fiber, and (c) $\Phi$ along $\eta$ fiber.

tures and grain size for all the test steels. As mentioned above, effective measures to reduce core loss of non-oriented electrical steel are needed to decrease the hysteresis loss that dominates total iron loss. ${ }^{6}$ In order to achieve this purpose, an optimum grain diameter is necessary for nonorientated electrical steel by optimizing manufacturing process. For all the test steels, grain grew significantly in two-step annealing and its growth increased by $8-14 \%$ comparing to one-step annealing. The Mo bearing steel revealed more growth rate than $\mathrm{Co}$ and $\mathrm{Ni}$ bearing steels. The Co steel showed lowest growth, which means that Co is most effective to restrict grain growth. It is obvious that these elements play a role to hinder recrystallization growth during both annealing methods as these steels are compared with the reference steel A which shows larger grain growth by two-step annealing. Furthermore, texture change is greatly important because texture is changed according to grain growth and it is very much related to magnetic properties. Development of cube and Goss textures in the re- crystallization process is beneficial to magnetic properties but $\gamma$ fiber is detrimental. Overall results in this work indicate that cube and Goss textures were slightly increased by two-step annealing comparing with single-step annealing. However, one distinct result turned out that $\gamma$ fiber of $\mathrm{Ni}$ steel was higher than that of Co and Mo steels after cold rolling but it became lower after both annealing methods, which implies that $\mathrm{Ni}$ element plays a role in reducing $\gamma$ fiber during annealing. Unfortunately it was not verified how Ni reduced $\gamma$ fiber in the present work.

In regard of relationship between texture factor and magnetic properties, higher texture factor generally results in lower iron loss and higher magnetic flux density. ${ }^{5,7)}$ However, in the present result, texture factor was not increased conspicuously by two-step annealing, as illustrated in Fig. 5. The overall results in both annealing processes showed that samples annealed in two-step revealed more or less similar $\alpha / / \mathrm{RD}$ fibers and $\gamma / / \mathrm{ND}$ fibers to single-step annealed materials (Fig. 4), which also results in a little in- 
Table 5. Magnetic properties of annealed sheets.

\begin{tabular}{|c|c|c|c|c|c|c|c|c|}
\hline \multirow{3}{*}{ Steels } & \multicolumn{9}{|c|}{ Magnetic properties } \\
\cline { 2 - 9 } & \multicolumn{3}{|c|}{ Magnetic flux density $\left(\mathrm{B}_{50}\right)$, Tesla } & \multicolumn{3}{c|}{ Iron loss $\left(\mathrm{W}_{15 / 50}\right), \mathrm{W} / \mathrm{Kg}$} \\
\cline { 2 - 10 } & BL & BT & Mean & $\begin{array}{c}\text { Anistropy } \\
(\Delta \mathrm{B}) \%\end{array}$ & $\mathrm{WL}$ & $\mathrm{WT}$ & Mean & \multicolumn{2}{c|}{$\begin{array}{c}\text { Anistropy } \\
(\Delta \mathrm{W}) \%\end{array}$} \\
\hline A-FA1 & 1.692 & 1.649 & 1.671 & 1.29 & 2.194 & 2.300 & 2.247 & 2.36 \\
\hline B-FA1 & 1.687 & 1.640 & 1.664 & 1.41 & 2.167 & 2.358 & 2.263 & 4.22 \\
\hline C-FA1 & 1.692 & 1.642 & 1.667 & 1.50 & 2.238 & 2.382 & 2.310 & 3.12 \\
\hline D-FA1 & 1.710 & 1.646 & 1.678 & 1.92 & 2.136 & 2.303 & 2.219 & 3.77 \\
\hline A-FA2 & 1.700 & 1.642 & 1.671 & 1.74 & 2.101 & 2.230 & 2.165 & 2.98 \\
\hline B-FA2 & 1.677 & 1.634 & 1.656 & 1.30 & 2.160 & 2.316 & 2.238 & 3.49 \\
\hline C-FA2 & 1.670 & 1.638 & 1.654 & 0.97 & 2.191 & 2.323 & 2.257 & 2.92 \\
\hline D-FA2 & 1.721 & 1.647 & 1.684 & 2.20 & 2.047 & 2.240 & 2.144 & 4.50 \\
\hline
\end{tabular}

Note:

FA1 is single-step annealing and FA2 two-step annealing.

$\mathrm{W}_{\mathrm{L}}$ is longitudinal core loss and $\mathrm{W}_{\mathrm{T}}$ is transverse core loss.

$\mathrm{B}_{\mathrm{L}}$ and $\mathrm{B}_{\mathrm{T}}$ are longitudinal and transverse magnetic flux density respectively.

$\Delta \mathrm{B}$ is magnetic flux density anisotropy expressed as $\left|\left(\mathrm{B}_{\mathrm{L}}-\mathrm{B}_{\mathrm{T}}\right)\right| /\left(\mathrm{B}_{\mathrm{L}}+\mathrm{B}_{\mathrm{T}}\right)$.

$\Delta \mathrm{W}$ is core loss anisotropy expressed as $\left|\left(\mathrm{W}_{\mathrm{L}}-\mathrm{W}_{\mathrm{T}}\right)\right|\left(\mathrm{W}_{\mathrm{L}}+\mathrm{W}_{\mathrm{T}}\right)$.

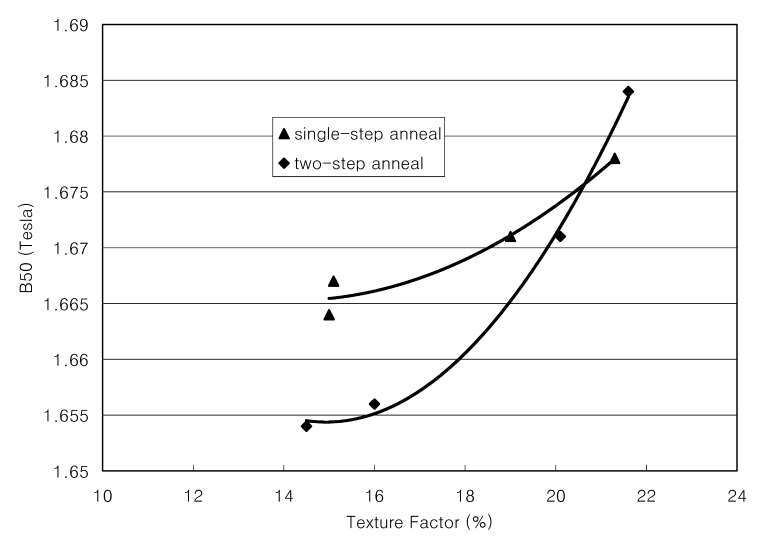

(a)

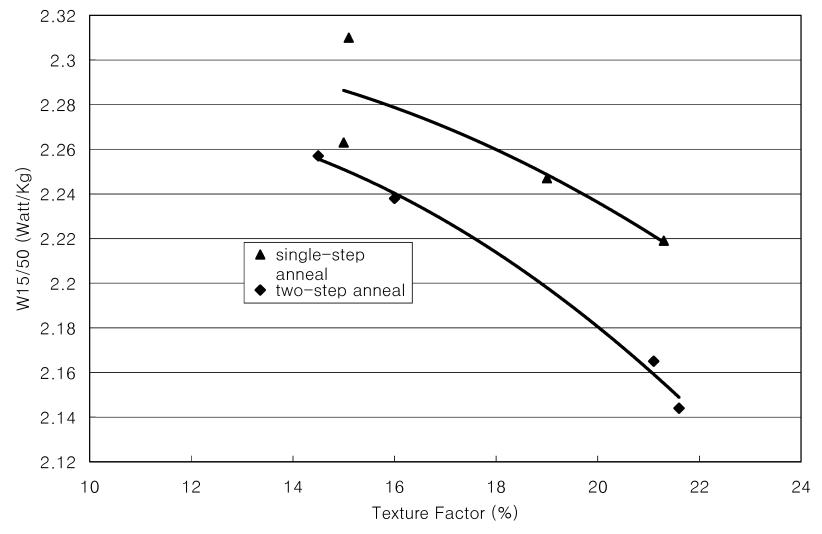

(b)

Fig. 5. Relationship between texture factor and (a) magnetic induction and (b) iron loss corresponding with annealing process.

crease of texture factor. Magnetic properties are usually concomitant with crystal texture, ${ }^{5)}$ i.e. cube and Goss textures which are favorable to magnetic properties, but $\gamma$ fiber is unfavorable. Even though two-step annealing did not promote the development of cube and Goss textures, it was evidenced that it promoted the reduction of iron loss. This im- provement of iron loss is supposed due to grain size increase in two-step annealing process, because grain boundary is detrimental to magnetic properties. Materials with larger grains have rather less number of total grain boundaries comparing to materials with smaller grains. So twostep annealing might have better effectiveness on lowering iron loss than single-step annealing due to the grain size effect. To begin with, two-step annealing was assumed to affect textures (texture factor) and thus magnetic properties. The answer to the assumption is found in Fig. 5, in which the magnetic flux density increased with increasing the magnetic factor for both annealing methods. A large texture factor means naturally that volume of cube texture and Goss texture is large comparing with $\gamma$ fiber. In case of twostep annealing, magnetic induction of $B_{50}$ is lower than onestep annealing up to texture factor of $20 \%$. The reason for this result is presumably thought that in regard of grain size effect, obtained from two-step annealing (Table 2), texture factor effect on $B_{50}$ between large and small grain under the same texture factor value become smaller for larger grain. That is, magnetic induction becomes lower for larger grain. However, if TF is over $20 \%$, that is, volume of cube and Goss textures is relatively more than $\gamma$ fiver, two-step annealing shows apparently higher magnetic induction than one-step annealing. On the other hand, in the relation between texture and iron loss, the latter decreases with an increase in texture factor and two-step annealing reveals lower iron loss level with texture factor than one-step annealing. This result is reverse to the magnetic flux density as mentioned above, of which reason is explained also by grain size effect, that is, two-step annealing brings about larger grains which result in a decrease in number of grain boundaries in a given volume of material and finally results in lowering iron loss.

Regarding effect of two-stage annealing on magnetic anisotropy, as though the effect of two-step annealing on magnetic anisotropy was different according to elements 
added as seen in Table 5, only Ni bearing steel showed large anisotropies of magnetic induction and iron loss in two-step annealing. It is evidenced from this result that Ni element plays a role to decreases iron loss and to enhance magnetic induction but to enlarge magnetic anisotropy.

From above discussion, it is concluded that two-step annealing is effective in lowering core loss and increasing magnetic induction due to reduction of $\gamma$ fiber and increase of cube texture.

\section{Conclusions}

In $3 \mathrm{Si}-1.4 \mathrm{Al}$ electrical steel with addition of $\mathrm{Co}$, Mo or $\mathrm{Ni}$, the texture of hot bands is almost random except $\gamma$ fiber which is dominant. After cold reduction, the texture shows the typical (111)//ND and (110)//RD fibers. When cold rolled sheets are annealed, it is clear that $\gamma$ fiber of $\{111\}\langle 110\rangle$ and $\{111\}\langle 112\rangle$ decreases significantly, while, on the contrary, Goss texture increases but cube texture is not changed. Two-step annealing process was observed to be effective to increase grain size and decrease $\gamma$ fiber texture resulting in lowering iron loss and its anisotropy. The combination of increasing grain size and decreasing $\gamma$ texture by two-step annealing was able to improve magnetic properties.

\section{REFERENCES}

1) B. Y. Huang, K. Yamamoto, C. Kaido and Y. Yamashiro: IEEE Tran. Magn., 35 (1999), No. 5, 3376.

2) A. J. Moses: IEEE Proc., 137 (1990), No. 5, 233.

3) G. Lyudkoushy, P. K. Rastogi and M. Bala: J. Met., 22 (1986), 18.

4) G. Lyudkovsky and P. D. Southwick: Metall. Trans. A, 17A (1986), 1287.

5) S. K. Chang: Mater. Sci., 41 (2006), 7380.

6) K. Honma, T. Nozawa and H. Kobayashi: IEEE. Tran. Magn., 21 (1985), No. 5, 1903.

7) S. K. Chang and W. Y. Huang: ISIJ Int., 45 (2005), No. 6, 918. 